\title{
Stieltjes constants of $L$-functions in the extended Selberg class
}

\author{
Shōta Inoue ${ }^{1}$. Sumaia Saad Eddin ${ }^{2}$ - Ade Irma Suriajaya ${ }^{3}$ (D)
}

Received: 22 August 2019 / Accepted: 11 January 2021 / Published online: 20 March 2021

(c) The Author(s) 2021

\begin{abstract}
Let $f$ be an arithmetic function and let $\mathcal{S}^{\#}$ denote the extended Selberg class. We denote by $\mathcal{L}(s)=\sum_{n=1}^{\infty} \frac{f(n)}{n^{s}}$ the Dirichlet series attached to $f$. The Laurent-Stieltjes constants of $\mathcal{L}(s)$, which belongs to $\mathcal{S}^{\#}$, are the coefficients of the Laurent expansion of $\mathcal{L}$ at its pole $s=1$. In this paper, we give an upper bound of these constants, which is a generalization of many known results.
\end{abstract}

Keywords Laurent-Stieltjes constant $\cdot L$-function $\cdot$ Extended Selberg class $\cdot$ Upper bound

Mathematics Subject Classification 11N37 · 11Y60

The first author is supported by Grant-in-Aid for JSPS Research Fellow (Grant No.: 19J11223). The second author is supported by the Austrian Science Fund (FWF): Project F5505-N26 and Project F5507-N26, which are parts of the special Research Program "Quasi Monte Carlo Methods: Theory and Application". The third author is supported by JSPS KAKENHI Grant Number 18K13400 and conducted a part of the research under the RIKEN Special Postdoctoral Researcher program as a member of iTHEMS.

$凶 \quad$ Ade Irma Suriajaya

adeirmasuriajaya@math.kyushu-u.ac.jp

Shōta Inoue

m16006w@math.nagoya-u.ac.jp

Sumaia Saad Eddin

sumaia.saad_eddin@jku.at

1 Graduate School of Mathematics, Nagoya University, Furo-cho, Chikusa-ku, Nagoya 464-8602, Japan

2 Institute of Financial Mathematics and Applied Number Theory, JKU Linz, Altenbergerstraße 69, 4040 Linz, Austria

3 Faculty of Mathematics, Kyushu University, 744 Motooka, Nishi-ku, Fukuoka 819-0395, Japan 


\section{Introduction}

Let $q$ be any positive integer $\geq 1$ and let $\chi$ be a Dirichlet character modulo $q$. Let $\gamma_{n}(\chi)$ denote the Laurent coefficients of the Dirichlet L-function $L(s, \chi)$ near $s=1$. We recall that

$$
\gamma_{n}(\chi)=\sum_{a=1}^{q} \chi(a) \gamma_{n}(a, q)
$$

where

$$
\gamma_{n}(a, q)=\lim _{M \rightarrow \infty} \sum_{1 \leq m \equiv a \bmod q}^{M} \frac{(\log m)^{n}}{m}-\frac{(\log M)^{n+1}}{q(n+1)}
$$

In particular, $\gamma_{0}(1,1)=0.5772156649 \cdots$ is the well-known Euler constant. The constants $\gamma_{n}(a, q)$ are often called the Stieltjes constants or generalized Euler constants. In the particular case when $\chi=\chi_{0}$, where $\chi_{0}$ is the principal character modulo 1 , the Dirichlet $L$-function $L\left(s, \chi_{0}\right)$ reduces to the Riemann zeta function $\zeta(s)$, that is $L\left(s, \chi_{0}\right)=\zeta(s)$. We write the corresponding Laurent coefficients simply $\gamma_{n}\left(\chi_{0}\right)=\gamma_{n}(1,1)=\gamma_{n}$. Stieltjes in 1885 showed that

$$
\gamma_{n}=\frac{(-1)^{n}}{n !} \lim _{M \rightarrow \infty}\left(\sum_{m=1}^{M} \frac{(\log m)^{n}}{m}-\frac{(\log M)^{n+1}}{(n+1)}\right),
$$

which pioneered the study of Laurent coefficients of zeta functions and $L$-functions. This gives rise to the widely used name "Stieltjes constants" for these coefficients.

The asymptotic behavior of $\gamma_{n}$ as $n \rightarrow \infty$ has been widely studied by many authors (for instance: Briggs [5], Mitrovic̀ [13], Israilov [10], Matsuoka [12], and more recently, Coffey [6,7], Knessl and Coffey [11], Adell [2], Adell and Lekuona [1], and Saad Eddin [16]). The studies mostly focused on the growth and sign changes of the sequence $\left(\gamma_{n}\right)$, explicit upper estimates for $\left|\gamma_{n}\right|$, and asymptotic expressions for $\gamma_{n}$. Stieltjes constants for other zeta functions and $L$-functions have also been studied by many authors. We introduce some of their results in the following section.

In this paper, we are interested in investigating the Stieltjes constants of more general $L$-functions. We consider functions in a class larger than the Selberg class. We first introduce the Selberg class $\mathcal{S}$.

\section{Selberg class}

Let $f$ be an arithmetic function, and denote by $\mathcal{L}(s)=\sum_{n=1}^{\infty} \frac{f(n)}{n^{s}}$ the Dirichlet series attached to $f$. We say the Dirichlet series $\mathcal{L}(s)$ belongs to the Selberg class $\mathcal{S}$ if it is absolutely convergent when $\operatorname{Re}(s)>1$ and satisfies the following properties:

Condition $\mathcal{S} 1$ Ramanujan hypothesis: For any $\varepsilon>0$, we have $f(n) \ll_{\varepsilon} n^{\varepsilon}$. 
Condition $\mathcal{S} 2$ Analytic continuation: There exists $k \in \mathbb{Z}_{\geq 0}$ such that $(s-1)^{k} \mathcal{L}(s)$ is entire of finite order.

Condition $\mathcal{S} 3$ Functional equation: Define

$$
\mathcal{F}(s):=Q^{s} \prod_{j=1}^{r} \Gamma\left(\lambda_{j} s+\mu_{j}\right),
$$

where $Q, \lambda_{i}$ are positive real numbers, $\Gamma$ is the gamma function, $\mu_{j}$ is a complex number satisfying $\operatorname{Re}\left(\mu_{j}\right) \geq 0$. Then the function $\Phi(s):=\mathcal{F}(s) \mathcal{L}(s)$ satisfies the functional equation

$$
\Phi(s)=\omega \overline{\Phi(1-\bar{s})}
$$

where $\omega$ is a complex number with $|\omega|=1$.

Condition $\mathcal{S} 4$ Euler product: For $\operatorname{Re}(s)>1$, the function $\mathcal{L}(s)$ can be written as a product over prime numbers $p$ :

$$
\mathcal{L}(s)=\prod_{p} \mathcal{L}_{p}(s),
$$

where

$$
\mathcal{L}_{p}(s)=\exp \left(\sum_{k=1}^{\infty} \frac{b\left(p^{k}\right)}{p^{k s}}\right),
$$

with $b(n) \ll n^{\theta}$, for some $\theta<\frac{1}{2}$.

All functions $\mathcal{L}$ in $\mathcal{S}$ are expected to satisfy the Riemann hypothesis. If we remove any of the above conditions, however, this is no longer true. In this particular case, the hypothesis is usually called the Grand Riemann Hypothesis: all non-trivial zeros of $\mathcal{L}(s) \in \mathcal{S}$ lie on $\operatorname{Re}(s)=1 / 2$. The extended Selberg class $\mathcal{S}^{\#}$ is defined to be the class of functions $\mathcal{L}(s)=\sum_{n=1}^{\infty} \frac{f(n)}{n^{s}}$ satisfying the above conditions $\mathcal{S} 2$ and $\mathcal{S} 3$, but not necessarily $\mathcal{S} 1$ and $\mathcal{S} 4$.

Notable examples of functions in $\mathcal{S}$ are the Riemann zeta function $\zeta(s)$, Dirichlet $L$-functions $L(s, \chi)$ associated with non-principal primitive characters $\chi$, and the Dedekind zeta function $\zeta_{K}(s)$ of a number field $K$. The sum of the parameters $\lambda_{j}$ in $\mathcal{S} 3$ gives the degree of the $L$-function $\mathcal{L}(s)$ in $\mathcal{S}^{\#}$, and so in $\mathcal{S}$, as follows:

$$
d_{\mathcal{L}}=2 \sum_{j=1}^{r} \lambda_{j}
$$

It is not known if $d_{\mathcal{L}} \in \mathbb{Z}_{>0}$ for all $\mathcal{L} \in \mathcal{S}$ but the degree $d_{\mathcal{L}}$ characterizes certain properties of the functions $\mathcal{L} \in \mathcal{S}$. Although the functional equation is not unique, the 
degree $d_{\mathcal{L}}$ is well defined and captures the growth of $\mathcal{L}$. We shall not discuss $d_{\mathcal{L}}$ further since it is irrelevant to the aim of this paper. The readers may refer to [19, Chap. 6] for more details about the Selberg class.

From now on we keep our focus on $\mathcal{L} \in \mathcal{S}^{\#}$. That is, we would like to extend beyond the Selberg class by eliminating conditions $\mathcal{S} 1$ and $\mathcal{S} 4$. Consider the Laurent expansion of $\mathcal{L}(s)$ at its possible pole $s=1$ written in the following form:

$$
\mathcal{L}(s)=\sum_{n=-k}^{\infty} \gamma_{n}(\mathcal{L})(s-1)^{n} .
$$

We call the coefficients $\gamma_{n}(\mathcal{L})$ the generalized Laurent-Stieltjes constants or the Laurent-Stieltjes constants of the extended Selberg class. In this paper, we study these coefficients and give an upper bound of $\gamma_{n}(\mathcal{L})$ for $\mathcal{L} \in \mathcal{S}^{\#}$.

Our main theorem is stated as follows.

Theorem Let $\mathcal{L} \in \mathcal{S}^{\#} \backslash\{0\}$ and let $d_{\mathcal{L}}$ be the degree of $\mathcal{L}$. Let $Q$ be the positive real number appearing in condition $\mathcal{S} 3$ and let

$$
\lambda_{m}:=\min _{1 \leq j \leq r} \lambda_{j}, \quad \lambda_{M}:=\max _{1 \leq j \leq r} \lambda_{j}, \quad \text { and } \quad \mu_{M}:=\max _{1 \leq j \leq r}\left|\mu_{j}\right| .
$$

For a positive integer $n$ with

$$
\frac{n}{\log n}>\left(\frac{1}{2}+\frac{\mu_{M}+1}{\lambda_{m}}\right) d_{\mathcal{L}} \log (Q+3),
$$

we have

$$
\left|\gamma_{n}(\mathcal{L})\right| \leq C_{\mathcal{L}}(a) a^{-n}\left(2+\frac{1}{n-\frac{d_{\mathcal{L}}(2 a-1)}{2}}\right)
$$

where a satisfies $1+\frac{\mu_{M}+1}{\lambda_{m}}<a<\frac{1}{2}+\frac{n}{d_{\mathcal{L}}}$ and

$$
\begin{aligned}
C_{\mathcal{L}}(a)= & \frac{2^{r} Q^{2 a-1}}{\pi} \exp \left(\frac{1}{5} \sum_{j=1}^{r} \frac{1}{\lambda_{j}(a-1)-\operatorname{Re}\left(\mu_{j}\right)}\right) \\
& \times\left(\sum_{m=1}^{\infty} \frac{|f(m)|}{m^{a}}\right)\left(8 \lambda_{M}^{2} a^{2}\right)^{\frac{d}{4}}(2 a-1)
\end{aligned}
$$

Finally we remark that the Laurent-Stieltjes constants of zeta and $L$-functions have many applications not only in analytic number theory, but also in algebraic number theory and even fields outside of number theory. They can be used to determine zerofree regions for $L(s, \chi)$ near the real axis in the critical strip $0 \leq \operatorname{Re}(s) \leq 1$, to compute the values of $\zeta(s)$ in the complex plane, to study the class number of a quadratic field, etc. Further, they appear in an explicit expression of Li's criterion which relates them to the Riemann hypothesis, see [4]. 


\section{Some known results on the Laurent-Stieltjes constants of zeta and L-functions}

The first explicit upper bound for $\left|\gamma_{n}\right|$ has been given by Briggs [5], which was later improved by Berndt [3] and Israilov [10]. In 1985, the theory made a huge progress via an asymptotic expansion shown by Matsuoka [12], for these constants. He gave an excellent upper bound of $\left|\gamma_{n}\right|$ for $n \geq 10$ and proved that

$$
\left|\gamma_{n}\right| \leq 10^{-4} e^{n \log \log n}
$$

This result had been the best upper bound of $\left|\gamma_{n}\right|$ for more than 20 years. Thanks to the above result, Matsuoka showed that $\zeta(s)$ has no zeros in the region $|s-1| \leq \sqrt{2}$, with $0 \leq \operatorname{Re}(s) \leq 1$. Many have tried to improve on the Matsuoka bound, with few successful attempts. Matsuoka's work relied on a formula that is essentially a consequence of Cauchy's integral theorem and the functional equation. More recently, the second author [16] extended this formula to Dirichlet $L$-functions. She gave the following upper bound for $\left|\gamma_{n}(\chi)\right|$ for primitive Dirichlet characters $\chi$ modulo $q$ and for every $1 \leq q \leq \pi e^{(n+1) / 2} /(2 n+2)$. We have

$$
\frac{\left|\gamma_{n}(\chi)\right|}{n !} \leq q^{-1 / 2} C(n, q) \min \left(1+D(n, q), \frac{\pi^{2}}{6}\right)
$$

where

$$
C(n, q)=2 \sqrt{2} \exp \left\{-(n+1) \log \theta(n, q)+\theta(n, q)\left(\log \theta(n, q)+\log \frac{2 q}{\pi e}\right)\right\}
$$

and

$$
\theta(n, q)=\frac{n+1}{\log \frac{2 q(n+1)}{\pi}}-1, \quad D(n, q)=2^{-\theta(n, q)-1} \frac{\theta(n, q)+1}{\theta(n, q)-1}
$$

In the case when $\chi=\chi_{0}$ and $q=1$, this leads to a sizable improvement of Matsuoka's bound and of previous results. As an application of this upper bound, the second author showed in [17] that this result enables us to approximate $L(s, \chi)$ in the neighborhood of $s=1$ by a short Taylor polynomial. For $N=4 \log q$ and $q \geq 150$, we have

$$
\left|L(s, \chi)-\sum_{n \leq N} \frac{(-1)^{n} \gamma_{n}(\chi)}{n !}(s-1)^{n}\right| \leq \frac{32.3}{q^{2.5}}
$$

where $|s-1| \leq e^{-1}$. She also proved that the function $\zeta(s)$ has no zeros in the region $|s-1| \leq 2.2093$ with $0 \leq \operatorname{Re}(s) \leq 1$. This result is an improvement of Matsuoka's result. 
Finally, let $K$ be a number field and $\mathcal{O}_{K}$ be its ring of integers. Define for $\operatorname{Re}(s)>1$ the Dedekind zeta function

$$
\zeta_{K}(s)=\sum_{\mathfrak{a}} \frac{1}{N \mathfrak{a}^{s}}=\prod_{\mathfrak{p}} \frac{1}{1-N \mathfrak{p}^{-s}}
$$

where a runs over non-zero ideals in $\mathcal{O}_{K}, \mathfrak{p}$ runs over the prime ideals in $\mathcal{O}_{K}$ and $N \mathfrak{a}$ is the norm of $\mathfrak{a}$. It is known that $\zeta_{K}(s)$ can be analytically continued to $\mathbb{C} \backslash\{1\}$, and that at $s=1$ it has a simple pole, with residue $\gamma_{-1}(K)$ given by the analytic class number formula:

$$
\gamma_{-1}(K)=\frac{2^{r_{1}}(2 \pi)^{r_{2}} h(K) R(K)}{\omega(K) \sqrt{|D(K)|}} .
$$

Here we denote by $r_{1}$ the number of real embeddings of $K, r_{2}$ the number of complex embeddings of $K, h(K)$ the class number of $K, R(K)$ the regulator of $K, \omega(K)$ the number of roots of unity contained in $K$ and $D(K)$ the discriminant of the extension $K / \mathbb{Q}$. Consider the Laurent expansion

$$
\zeta_{K}(s)=\frac{\gamma_{-1}(K)}{s-1}+\sum_{n=0}^{\infty} \gamma_{n}(K)(s-1)^{n}
$$

of $\zeta_{K}(s)$ at $s=1$. The constants $\gamma_{n}(K)$ are sometimes called the Stieltjes constants associated with the Dedekind zeta function. In [8] they are called higher Euler's constants of $K$. The second author [18] studied these constants and showed that, for $n \geq 1$, we have

$$
\gamma_{n}(K)=\frac{(-1)^{n}}{n !} \lim _{x \rightarrow \infty}\left(\sum_{N \mathfrak{a} \leq x} \frac{(\log N \mathfrak{a})^{n}}{N \mathfrak{a}}-\gamma_{-1}(K) \frac{(\log x)^{n+1}}{n+1}\right)
$$

and

$$
\gamma_{0}(K)=\lim _{x \rightarrow \infty}\left(\sum_{N \mathfrak{a} \leq x} \frac{1}{N \mathfrak{a}}-\gamma_{-1}(K) \log x\right)+\gamma_{-1}(K)
$$

To conclude this section, we remark that only the first constant $\gamma_{K}=\gamma_{0}(K) / \gamma_{-1}(K)$, called the Euler-Kronecker constant, which is closely related to values of the logarithmic derivative of $L$-functions, has been studied so far. For more details see for example $[9,14,20]$. This raises questions on the other Stieltjes constants associated with $\zeta_{K}(s)$. The authors were motivated to give partial answers to these questions in a much more general context, that is, for all $L$-functions in the extended Selberg class. 


\section{Auxiliary lemmas}

In order to prove our main result, we first show a proposition and two necessary lemmas. Recall the notation used when we defined $\mathcal{S}^{\#}$ in Sect. 1.

Lemma 1 Let $\mathcal{L} \in \mathcal{S}^{\#}$ and let $d_{\mathcal{L}}$ be the degree of $\mathcal{L}$. Then we have

$$
\mathcal{L}(\sigma+i t) \asymp \mathcal{L}|t|^{d_{\mathcal{L}}\left(\frac{1}{2}-\sigma\right)}|\mathcal{L}(1-\sigma+i t)|
$$

as $|t| \rightarrow \infty$. In particular,

$$
\mathcal{L}(\sigma+i t) \ll_{\mathcal{L}, \varepsilon} \begin{cases}|t|^{\varepsilon} & \text { if } \sigma \geq 1, \\ |t|^{\frac{1-\sigma}{2} d_{\mathcal{L}}+\varepsilon} & \text { if } 0 \leq \sigma \leq 1, \\ |t|^{\left(\frac{1}{2}-\sigma\right) d_{\mathcal{L}+\varepsilon}} & \text { if } \sigma \leq 0 .\end{cases}
$$

Proof For the standard case when we assume $\mathcal{S} 1$, see [19, Theorem 6.8]. Note here that the first-half (2) is obtained from the functional equation $\mathcal{S} 3$.

Now without $\mathcal{S} 1$, we note that the absolute convergence of the Dirichlet series and partial summation imply for any $\varepsilon>0$,

$$
\sum_{n \leq x}|f(n)| \ll_{\varepsilon} x^{1+\varepsilon}
$$

Note that this is weaker than $\mathcal{S} 1$, but is sufficient for our purpose. Using (4) in combination with the functional equation $\mathcal{S} 3$, we can easily show the bounds (3) for the case $\sigma \geq 1+\varepsilon$ and $\sigma \leq-\varepsilon$. More precisely,

$$
\mathcal{L}(\sigma+i t) \ll_{\mathcal{L}, \varepsilon} \begin{cases}1 & \text { if } \sigma \geq 1+\varepsilon, \\ |t|^{\left(\frac{1}{2}-\sigma\right) d_{\mathcal{L}}+\varepsilon} & \text { if } \sigma \leq-\varepsilon .\end{cases}
$$

Since the function $\mathcal{L}(s)$ is entire of finite order from condition $\mathcal{S} 2$, for any $\delta>0$,

$$
\mathcal{L}(\sigma+i t) \ll_{\mathcal{L}, \varepsilon} \exp \exp (\delta|t|)
$$

holds in the strip $-1 \leq \sigma \leq 2$. Substituting this into (2), we can show that this also holds for $0 \leq \sigma \leq 1 / 2$. Applying the theorem of Phragmén-Lindelöf [15, Proposition 8.15], we have

$$
\mathcal{L}(\sigma+i t) \ll \mathcal{L}, \varepsilon_{\varepsilon}|t|^{\frac{1-\sigma}{2} d_{\mathcal{L}}+\varepsilon}
$$

for $0 \leq \sigma \leq 1$

Proposition 1 Let $\mathcal{L} \in \mathcal{S}^{\#}$ with degree $d_{\mathcal{L}}>0$. Let $n$ be an integer with $n>$ $\max \left\{0, \frac{d_{\mathcal{L}}}{2}-1\right\}$. For $1<a<\frac{n+1}{d_{\mathcal{L}}}+\frac{1}{2}$ such that $\lambda_{j}(1-a)+\operatorname{Re}\left(\mu_{j}\right) \notin \mathbb{Z}$ for 
each $j=1,2, \ldots, r$, we have

$$
\gamma_{n}(\mathcal{L})=\frac{(-1)^{n}}{2 \pi i} \int_{a-i \infty}^{a+i \infty} \frac{G_{\mathcal{L}}(s)}{s^{n+1}} \overline{\mathcal{L}(\bar{s})} \mathrm{d} s
$$

where the function $G_{\mathcal{L}}$ is defined by

$$
G_{\mathcal{L}}(s):=\frac{\omega Q^{2 s-1}}{\pi^{r}} \prod_{j=1}^{r} \Gamma\left(\lambda_{j} s+\bar{\mu}_{j}\right) \sin \left(\pi\left(\lambda_{j}(1-s)+\mu_{j}\right)\right) \Gamma\left(\lambda_{j}(s-1)+1-\mu_{j}\right) .
$$

Here $Q, \lambda_{i}$ are positive real numbers, $\mu_{j}$ and $\omega$ are complex numbers with $\operatorname{Re}\left(\mu_{j}\right) \geq 0$ and $|\omega|=1$.

Proof By Cauchy's formula, we can write

$$
\gamma_{n}(\mathcal{L})=\frac{1}{2 \pi i} \int_{D} \frac{\mathcal{L}(s)}{(s-1)^{n+1}} \mathrm{~d} s
$$

where $D$ is the positively oriented rectangular path passing through the vertices $-a+$ $1+i T,-a+1-i T, A-i T$ and $A+i T$, where $A$ and $T$ are sufficiently large numbers. Let us now divide $D$ into the line segments $D_{1}, D_{2}, D_{3}$ and $D_{4}$ joining $-a+1+i T$, $-a+1-i T, A-i T, A+i T$ and $-a+1+i T$, as in Fig. 1. Then, we have

$$
\gamma_{n}(\mathcal{L})=\frac{1}{2 \pi i}\left(\int_{D_{1}}+\int_{D_{2}}+\int_{D_{3}}+\int_{D_{4}}\right) \frac{\mathcal{L}(s)}{(s-1)^{n+1}} \mathrm{~d} s .
$$

By Lemma 1, the integral over $D_{2}$ is bounded by

$$
\begin{aligned}
\left|\int_{D_{2}} \frac{\mathcal{L}(s)}{(s-1)^{n+1}} \mathrm{~d} s\right|= & \left|\left(\int_{-a+1-i T}^{-i T}+\int_{-i T}^{1-i T}+\int_{1-i T}^{A-i T}\right) \frac{\mathcal{L}(s)}{(s-1)^{n+1}} \mathrm{~d} s\right| \\
& \ll \mathcal{L}, \varepsilon \\
& \times\left(\int_{-a+1}^{-n-1+\varepsilon} T^{d_{\mathcal{L}}\left(\frac{1}{2}-\sigma\right)} \mathrm{d} \sigma+\int_{0}^{1} T^{\frac{1}{2} d_{\mathcal{L}}(1-\sigma)} \mathrm{d} \sigma+\int_{1}^{A} \mathrm{~d} \sigma\right) \\
& \ll_{\mathcal{L}, \varepsilon} T^{-n-1+d_{\mathcal{L}}(a-1 / 2)+\varepsilon} .
\end{aligned}
$$

Since $a<\frac{n+1}{d_{\mathcal{L}}}+\frac{1}{2}$, the last term vanishes as $T \rightarrow+\infty$. Therefore, the integral over $D_{2}$ tends to 0 as $T \rightarrow+\infty$. A similar argument shows that the integral over $D_{4}$ tends to 0 as $T \rightarrow+\infty$.

Next we consider the integral over $D_{3}$. For $n>0$, we find that

$$
\left|\int_{D_{3}}\right| \ll_{\mathcal{L}, n} \int_{0}^{\infty} \frac{\mathrm{d} t}{\left((A-1)^{2}+t^{2}\right)^{(n+1) / 2}}<+\infty
$$




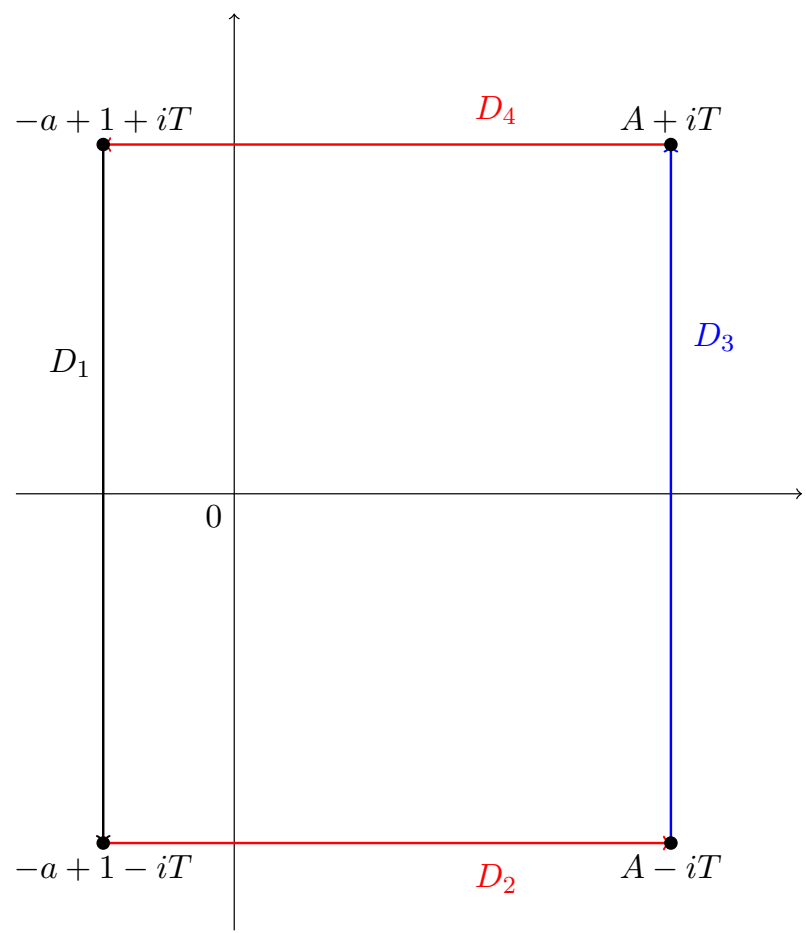

Fig. 1 The rectangle $D$ in the complex $s$ plane

and for any $t \geq 0$,

$$
\lim _{A \rightarrow+\infty} \frac{1}{\left((A-1)^{2}+t^{2}\right)^{(n+1) / 2}}=0 .
$$

Hence by Lebesgue's convergence theorem, we have $\lim _{A \rightarrow+\infty} \lim _{T \rightarrow+\infty} \int_{D_{3}}=0$.

Thus, for $n>\max \left\{0, \frac{d_{\mathcal{L}}}{2}-1\right\}$, we have

$$
\gamma_{n}(\mathcal{L})=\frac{1}{2 \pi i} \int_{-a+1+i \infty}^{-a+1-i \infty} \frac{\mathcal{L}(s)}{(s-1)^{n+1}} \mathrm{~d} s=\frac{(-1)^{n}}{2 \pi i} \int_{a-i \infty}^{a+i \infty} \frac{\mathcal{L}(1-s)}{s^{n+1}} \mathrm{~d} s .
$$

Here, by using the functional equation $\mathcal{S} 3$ for $\mathcal{L}(s)$ and the formula $\Gamma(s) \Gamma(1-$ $s) \sin (\pi s)=\pi$, we have

$$
\begin{aligned}
\mathcal{L}(1-s) & =\overline{\mathcal{L}(\bar{s})}\left(\omega \frac{\overline{\mathcal{F}(\bar{s})}}{\mathcal{F}(1-s)}\right)=\overline{\mathcal{L}(\bar{s})}\left(\omega Q^{2 s-1} \prod_{j=1}^{r} \frac{\Gamma\left(\lambda_{j} s+\overline{\mu_{j}}\right)}{\Gamma\left(\lambda_{j}(1-s)+\mu_{j}\right)}\right) \\
& =\overline{\mathcal{L}(\bar{s})} G_{\mathcal{L}}(s) .
\end{aligned}
$$


Hence

$$
\gamma_{n}(\mathcal{L})=\frac{(-1)^{n}}{2 \pi i} \int_{a-i \infty}^{a+i \infty} \frac{G_{\mathcal{L}}(s)}{s^{n+1}} \overline{\mathcal{L}(\bar{s})} \mathrm{d} s
$$

where the function $G_{\mathcal{L}}(s)$ is as defined in (5). This completes the proof of Proposition 1.

Lemma 2 For $\mathcal{L} \in \mathcal{S}^{\#} \backslash\{0\}$, consider $G_{\mathcal{L}}$ as defined in Proposition 1. Let $\lambda_{m}:=\min _{1 \leq j \leq r} \lambda_{j}$ and $\mu_{M}:=\max _{1 \leq j \leq r}\left|\mu_{j}\right|$. For $a>1+\frac{\mu_{M}}{\lambda_{m}}$, we have

$$
\left|G_{\mathcal{L}}(a+i t)\right| \leq c_{\mathcal{L}}(a) Q^{2 a-1}\left(\left(a \lambda_{M}+\mu_{M}+1\right)^{2}+\left(\lambda_{M}|t|+\mu_{M}\right)^{2}\right)^{\frac{d_{\mathcal{L}}}{4}(2 a-1)}
$$

where the constant $c_{\mathcal{L}}(a)$ is defined by

$$
c_{\mathcal{L}}(a)=2^{r} \exp \left(\frac{1}{5} \sum_{j=1}^{r} \frac{1}{\lambda_{j}(a-1)-\operatorname{Re}\left(\mu_{j}\right)}\right)
$$

Proof Put $\lambda_{m}:=\min _{1 \leq j \leq r} \lambda_{j}, \lambda_{M}:=\max _{1 \leq j \leq r} \lambda_{j}, \mu_{M}:=\max _{1 \leq j \leq r}\left|\mu_{j}\right|$, and let $a>$ $1+\frac{\mu_{M}}{\lambda_{m}}$. From (5), we have

$$
\begin{aligned}
\left|G_{\mathcal{L}}(a+i t)\right| \leq & \frac{Q^{2 a-1}}{\pi^{r}} \prod_{j=1}^{r} \mid \Gamma\left(\lambda_{j}(a+i t)+\overline{\mu_{j}}\right) \Gamma\left(\lambda_{j}(a-1+i t)\right. \\
& \left.+1-\mu_{j}\right) \sin \left(\pi\left(\lambda_{j}(1-a-i t)+\mu_{j}\right)\right) \mid .
\end{aligned}
$$

We can easily show that

$$
\left|\sin \left(\pi\left(\lambda_{j}(1-a-i t)+\mu_{j}\right)\right)\right| \leq \exp \left(\pi\left|\lambda_{j} t-\operatorname{Im}\left(\mu_{j}\right)\right|\right) .
$$

On the other hand, using Stirling's formula we can show that, for $x>0$,

$$
\begin{aligned}
\log |\Gamma(x+i y)|= & \frac{1}{2}\left(x-\frac{1}{2}\right) \log \left(x^{2}+y^{2}\right)-y \arctan (y / x)-x \\
& +\frac{1}{2} \log 2 \pi+\varphi(x, y) \\
& \leq \frac{1}{2}\left(x-\frac{1}{2}\right) \log \left(x^{2}+y^{2}\right)-\frac{\pi}{2}|y|+\frac{1}{2} \log 2 \pi+\varphi(x, y),
\end{aligned}
$$

where the function $\varphi(x, y)$ satisfies the inequality (cf. Binet's first formula)

$$
|\varphi(x, y)| \leq\left|\int_{0}^{\infty}\left(\frac{1}{2}-\frac{1}{t}+\frac{1}{e^{t}-1}\right) \frac{e^{-t(x+i y)}}{t} \mathrm{~d} t\right| \leq \frac{1}{10 x}
$$


From these inequalities, we find that (note that $\operatorname{Re}\left(\lambda_{j}(a-1+i t)+1-\mu_{j}\right) \geq 0$ since $\left.a>1+\mu_{M} / \lambda_{m}\right)$

$$
\begin{aligned}
& \left|\Gamma\left(\lambda_{j}(a+i t)+\overline{\mu_{j}}\right) \Gamma\left(\lambda_{j}(a-1+i t)+1-\mu_{j}\right) \sin \left(\pi\left(\lambda_{j}(1-a-i t)+\mu_{j}\right)\right)\right| \\
& \quad \leq 2 \pi \exp \left(\frac{1}{5\left(\lambda_{j}(a-1)-\operatorname{Re}\left(\mu_{j}\right)\right)}\right) \\
& \quad \times\left(\left(a \lambda_{M}+\mu_{M}+1\right)^{2}+\left(\lambda_{M}|t|+\mu_{M}\right)^{2}\right)^{\frac{\lambda_{j}}{2}(2 a-1)} .
\end{aligned}
$$

Hence we have

$$
\begin{aligned}
& \frac{Q^{2 a-1}}{\pi^{r}} \prod_{j=1}^{r} \mid \Gamma\left(\lambda_{j}(a+i t)+\overline{\mu_{j}}\right) \Gamma\left(\lambda_{j}(a-1+i t)\right. \\
& \left.\quad+1-\mu_{j}\right) \sin \left(\pi\left(\lambda_{j}(1-a-i t)+\mu_{j}\right)\right) \mid \\
& \quad \leq c_{\mathcal{L}}(a) Q^{2 a-1}\left(\left(a \lambda_{M}+\mu_{M}+1\right)^{2}+\left(\lambda_{M}|t|+\mu_{M}\right)^{2}\right)^{\frac{d_{\mathcal{L}}}{4}(2 a-1)},
\end{aligned}
$$

which completes the proof.

\section{Proof of Theorem}

Now we are ready to prove our main theorem. We again put

$$
\lambda_{m}:=\min _{1 \leq j \leq r} \lambda_{j}, \quad \lambda_{M}:=\max _{1 \leq j \leq r} \lambda_{j}, \quad \mu_{M}:=\max _{1 \leq j \leq r}\left|\mu_{j}\right|,
$$

and let $a$ be a real number satisfying $1+\frac{\mu_{M}+1}{\lambda_{m}}<a<\frac{1}{2}+\frac{n}{d_{\mathcal{L}}}$.

By Proposition 1 and Lemma 2, we have

$$
\begin{aligned}
\left|\gamma_{n}(\mathcal{L})\right| \leq & \frac{1}{2 \pi} \sum_{m=1}^{\infty} \frac{|f(m)|}{m^{a}} \int_{-\infty}^{\infty} \frac{\left|G_{\mathcal{L}}(a+i t)\right|}{\left(a^{2}+t^{2}\right)^{(n+1) / 2}} \mathrm{~d} t \\
\leq & \frac{c_{\mathcal{L}}(a)}{\pi} Q^{2 a-1} \sum_{m=1}^{\infty} \frac{|f(m)|}{m^{a}} \int_{0}^{\infty} \\
& \times \frac{\left(\left(a \lambda_{M}+\mu_{M}+1\right)^{2}+\left(\lambda_{M} t+\mu_{M}\right)^{2}\right)^{\frac{d_{\mathcal{L}}}{4}(2 a-1)}}{\left(a^{2}+t^{2}\right)^{(n+1) / 2}} \mathrm{~d} t,
\end{aligned}
$$

where

$$
c_{\mathcal{L}}(a)=2^{r} \exp \left(\frac{1}{5} \sum_{j=1}^{r} \frac{1}{\lambda_{j}(a-1)-\operatorname{Re}\left(\mu_{j}\right)}\right) .
$$


We divide the region of integration into two as follows:

$$
\left(\int_{0}^{A}+\int_{A}^{\infty}\right) \frac{\left(\left(a \lambda_{M}+\mu_{M}+1\right)^{2}+\left(\lambda_{M} t+\mu_{M}\right)^{2}\right)^{\frac{d \mathcal{L}}{4}(2 a-1)}}{\left(a^{2}+t^{2}\right)^{(n+1) / 2}} \mathrm{~d} t=: J_{1}+J_{2}
$$

with $A=a+\frac{1}{\lambda_{M}}$. We estimate $J_{1}$ and $J_{2}$ in the following manner:

$$
\begin{aligned}
J_{1} & \leq 2^{\frac{3}{4} d_{\mathcal{L}}(2 a-1)} \int_{0}^{A} \frac{\left(a \lambda_{M}\right)^{\frac{d_{\mathcal{L}}}{2}(2 a-1)}}{a^{n+1}} \mathrm{~d} t \leq\left(8 \lambda_{M}^{2}\right)^{\frac{d_{\mathcal{L}}}{4}(2 a-1)} \frac{A}{a} a^{-n+\frac{d_{\mathcal{L}}}{2}(2 a-1)} \\
& \leq 2\left(8 \lambda_{M}^{2}\right)^{\frac{d_{\mathcal{L}}}{4}(2 a-1)} a^{-n+\frac{d_{\mathcal{L}}}{2}(2 a-1)}
\end{aligned}
$$

and

$$
\begin{aligned}
J_{2} & \leq 2^{\frac{3}{4} d_{\mathcal{L}}(2 a-1)} \int_{A}^{\infty} \frac{\left(\lambda_{M} t\right)^{\frac{d_{\mathcal{L}}}{2}(2 a-1)}}{t^{n+1}} \mathrm{~d} t \leq\left(8 \lambda_{M}^{2}\right)^{\frac{d_{\mathcal{L}}}{4}(2 a-1)} \int_{A}^{\infty} \frac{\mathrm{d} t}{t^{n+1-d_{\mathcal{L}}(2 a-1) / 2}} \\
& \leq \frac{\left(8 \lambda_{M}^{2}\right)^{\frac{d_{\mathcal{L}}}{4}(2 a-1)}}{n-d_{\mathcal{L}}(2 a-1) / 2} a^{-n+\frac{d_{\mathcal{L}}}{2}(2 a-1)} .
\end{aligned}
$$

Substituting the above into (7), we obtain

$$
\begin{aligned}
\left|\gamma_{n}(\mathcal{L})\right| \leq & \frac{c_{\mathcal{L}}(a)}{\pi} Q^{2 a-1} \\
& \times\left(\sum_{m=1}^{\infty} \frac{|f(m)|}{m^{a}}\right) \\
& \times\left(8 \lambda_{M}^{2}\right)^{\frac{d \mathcal{L}}{4}(2 a-1)} a^{-n+\frac{d \mathcal{L}}{2}(2 a-1)}\left(2+\frac{1}{n-\frac{d_{\mathcal{L}}(2 a-1)}{2}}\right) .
\end{aligned}
$$

Therefore putting

$$
\begin{aligned}
C_{\mathcal{L}}(a)= & \frac{2^{r} Q^{2 a-1}}{\pi} \exp \\
& \times\left(\frac{1}{5} \sum_{j=1}^{r} \frac{1}{\lambda_{j}(a-1)-\operatorname{Re}\left(\mu_{j}\right)}\right)\left(\sum_{m=1}^{\infty} \frac{|f(m)|}{m^{a}}\right) \\
& \times\left(8 \lambda_{M}^{2} a^{2}\right)^{\frac{d_{\mathcal{L}}}{4}(2 a-1)}
\end{aligned}
$$

for $1+\frac{\mu_{M}+1}{\lambda_{m}}<a<\frac{1}{2}+\frac{n}{d_{\mathcal{L}}}$, we obtain

$$
\left|\gamma_{n}(\mathcal{L})\right| \leq C_{\mathcal{L}}(a) a^{-n}\left(2+\frac{1}{n-\frac{d_{\mathcal{L}}(2 a-1)}{2}}\right),
$$

which completes the proof of our Theorem. 
Open Access This article is licensed under a Creative Commons Attribution 4.0 International License, which permits use, sharing, adaptation, distribution and reproduction in any medium or format, as long as you give appropriate credit to the original author(s) and the source, provide a link to the Creative Commons licence, and indicate if changes were made. The images or other third party material in this article are included in the article's Creative Commons licence, unless indicated otherwise in a credit line to the material. If material is not included in the article's Creative Commons licence and your intended use is not permitted by statutory regulation or exceeds the permitted use, you will need to obtain permission directly from the copyright holder. To view a copy of this licence, visit http://creativecommons.org/licenses/by/4.0/.

\section{References}

1. Adell, J.A., Lekuona, A.: Fast computation of the Stieltjes constants. Math. Comput. 86, 2479-2492 (2017)

2. Adell, J.A.: Asymptotic estimates for Stieltjes constants: a probabilistic approach, Proc. R. Soc. Lond. Ser. A Math. Phys. Eng. Sci. 467, 954-963 (2011)

3. Berndt, B.C.: On the Hurwitz zeta-function. Rocky Mt. J. Math. 2(1), 151-157 (1972)

4. Bombieri, Enrico, Lagarias, Jeffrey C.: Complements to Li's criterion for the Riemann hypothesis. J. Number Theory 77(2), 274-287 (1999)

5. Briggs, W.E.: Some constants associated with the Riemann zeta-function, Mich. Math. J. 3, 117-121 (1955-1956)

6. Coffey, M.W.: Hypergeometric summation representations of the Stieltjes constants. Analysis (Munich) 33, 121-142 (2013)

7. Coffey, M.W.: Series representations for the Stieltjes constants. Rocky Mt. J. Math. 44, 443-477 (2014)

8. Hashimoto, Y., Iijima, Y., Kurokawa, N., Wakayama, M.: Euler's constants for the Selberg and the Dedekind zeta functions. Bull. Belg. Math. Soc. 11, 493-516 (2004)

9. Ihara, Y.: On the Euler-Kronecker constants of global fields and primes with small norms. In: Ginzburg, V. (ed.) Algebraic Geometry and Number Theory. In Honor of Vladimir Drinfeld's 50th Birthday, Progress in Mathematics, vol. 850, pp. 407-451. Birkhäuser Boston, Cambridge, MA (2006)

10. Israilov, M.I.: The Laurent expansion of the Riemann zeta function (in Russian). Mat. Inst. Steklova 158, 98-104 (1981)

11. Knessl, C., Coffey, M.W.: An effective asymptotic formula for the Stieltjes constants. Math. Comp. 80, 379-386 (2011)

12. Matsuoka, Y.: Euler, Generalized, constants associated with the Riemann zeta function, Number Theory and Combinatorics. Japan, : (Tokyo, Okayama and Kyoto, 1984), pp. 279-295. World Scientific Publishing, Singapore (1984)

13. Mitrović, D.: The signs of some constants associated with the Riemann zeta-function. Mich. Math. J 9, 395-397 (1962)

14. Moree, P.: Irregular Behaviour of Class Numbers and Euler-Kronecker Constants of Cyclotomic Fields: The Log Log Log Devil at Play. In: Pintz, J., Rassias, M. (eds.) Irregularities in the Distribution of Prime Numbers. Springer, Cham (2018)

15. Overholt, M.: A Course in Analytic Number Theory, Graduate Studies in Mathematics, vol. 160. American Mathematical Society, Providence, RI (2014)

16. Saad Eddin, S.: Explicit upper bounds for the Stieltjes constants, J. Number Theory 133, 1027-1044 (2013)

17. Saad Eddin, S.: Applications of the Laurent-Stieltjes constants for Dirichlet L-series. Proc. Jpn. Acad. Ser. A 93, 120-123 (2017)

18. Saad Eddin., S.: The signs of the Stieltjes constants associated with the Dedekind zeta-function. Proc. Jpn. Acad. 94(10), 93-96 (2018)

19. Steuding, J.: Value-Distribution of $L$-Functions. Lecture Notes in Mathematics, vol. 1877. Springer, Berlin (2007). IX+317pp

20. Tsfasman, M.A.: Asymptotic behaviour of the Euler-Kronecker constant. In: Ginzburg, V. (ed.) Algebraic Geometry and Number Theory. In Honor of Vladimir Drinfeld's 50th Birthday, Progress in Mathematics, vol. 850, pp. 453-458. Birkhäuser Boston, Cambridge, MA (2006)

Publisher's Note Springer Nature remains neutral with regard to jurisdictional claims in published maps and institutional affiliations. 